\title{
Highly efficient detection in fluorescence tomography of quantum dots using time-gated acquisition and ultrafast pulsed laser
}

\author{
Xiaofeng Zhang*, Cristian T. Badea \\ Center for In Vivo Microscopy, Duke University Medical Center, Durham, NC, 27710
}

\begin{abstract}
Quantum dots (QDs) are widely used in fluorescence tomography due to its unique advantages. Despite the very high quantum efficiency of the QDs, low fluorescent signal and autofluorescence are the most fundamental limitations in optical data acquisition. These limitations are particularly detrimental to image reconstruction for animal imaging, e.g., free-space in vivo fluorescence tomography. In animals studies, fluorescent emission from exogenous fluorescent probes (e.g. QDs) cannot be effectively differentiated from endogenous broad-spectral substances (mostly proteins) using optical filters. In addition, a barrow-band fluorescent filter blocks the majority of the fluorescent light and thus makes signal acquisition very inefficient. We made use of the long fluorescent lifetime of the QDs to reject the optical signal due to the excitation light pulse, and therefore eliminated the need for a fluorescent filter during acquisition. Fluorescent emission from the QDs was excited with an ultrafast pulsed laser, and was detected using a time-gated image intensifier. A tissue-simulating imaging phantom was used to validate the proposed method. Compared to the standard acquisition method that uses a narrow-band fluorescent filter, the proposed method is significantly more efficient in data acquisition (by a factor of $>10$ in terms of fluorescent signal intensity) and demonstrated reduction in autofluorescence. No additional imaging artifact was observed in the tomographic reconstruction.
\end{abstract}

Keywords: fluorescence tomography, time-gate acquisition, quantum dot

\section{INTRODUCTION}

Fluorescence tomography is a powerful imaging technology for studying biological processes in live organisms by means of interrogating intrinsic or extrinsic biomarkers. With the rapid development of new optical molecular probes, targeted fluorescence tomography becomes feasible in small animal in vivo imaging [1-3]. Using this imaging technology, a particular hypothesis for a given disease model can be noninvasively verified in live animals [4-6].

Among the conjugatable fluorescent markers, quantum dots (QDs) are excellent candidates for in vivo fluorescence tomography. QDs are semiconductor nano-crystals that have discrete energy transitions due to their three-dimensional confinement of electrons and holes. Compared to organic fluorophores, QDs have very unique photo-physical properties, such as high quantum efficiency, broad excitation spectrum, high photostability, long fluorescent lifetime, conjugatable to a broad variety of molecules, and a narrow emission spectrum tunable by particle size and composition [7, 8]. These properties make QDs a favorable choice for biomedical imaging, particularly for in vivo molecular imaging [9-11].

Due to the limited number of receptors for highly specific molecular probes, the amount of detectable fluorescent signal emitted from deep tissue is typically very low. In addition, although autofluorescence is relatively low in the near infrared spectrum compared to visible and ultraviolet, the very broad emission spectra of autofluorescence makes it ineffective to separate low fluorescent signals directly using optical filters. Several research groups have reported new methods using other contrast mechanisms, such as the spectral and temporal properties of the fluorophores, particularly multispectral and time-resolved methods $[5,12,13]$. These methods typically either require scanning the emission spectra (multispectral method) or the temporal response (time-resolved method) of the animal in addition to spatial scanning. As a result, the required data acquisition time is significantly prolonged due to the additional dimension of measurement. In addition, because the signal-to-noise ratios (SNR) of the measurements are reduced as a direct consequence of the narrowed acquisition bandwidth (either spectral or temporal), further increment of the acquisition time is typically required.

Fluorescent contrast can also be created based on the differences in the temporal dynamics of the excitation and the emission light, using a pulsed light source and time-gated photodetector. The concept of using time-gating to suppress

Optical Tomography and Spectroscopy of Tissue IX,

Edited by Bruce J. Tromberg, Arjun G. Yodh, Mamoru Tamura, Eva M. Sevick-Muraca, Robert R. Alfano, Proc. of SPIE Vol. 7896, 78962W - @ 2011 SPIE · CCC code: 1605-7422/11/\$18 - doi: 10.1117/12.875502 
autofluorescence in fluorescence measurements has been previously reported in 2D imaging of QDs in liquid samples, cells, or subcutaneous tumors in mice, in which fluorescent filters were still necessary [14-17]. We extended this timegated acquisition technique to 3D fluorescence tomography, which exploits not only the temporal characteristics of the fluorescent signals, but also that of the excitation light pulse, in order to more efficiently distinguish the fluorophore from its background environment. We used tissue-simulating imaging phantoms to demonstrate the proposed method. Near infrared light-emitting QDs were used as the fluorophore because of their unique advantages for biomedical imaging. Using the proposed acquisition method, the need for a fluorescent filter was eliminated.

\section{METHODS}

\subsection{Time-gated acquisition}

In time-resolved measurements, the fluorophore is excited by a pulse of light and emits fluorescent light. The temporal response of fluorescent emission is dictated by the relaxation rate of the molecules from the excited state to the ground state. If the relaxation rate is proportional to the population of excited molecules

$$
d N=-(N / \tau) d t
$$

the temporal response of a fluorophore excited by a short (instantaneous) excitation pulse is a mono-exponential function, and takes the form of

$$
s=s_{0} \exp (-t / \tau)=s_{0} \exp (-R t)
$$

where $N$ is the population of excited molecules, $s_{0}$ is the initial fluorescent signal intensity, $t$ is the time-delay relative to onset of the excitation pulse, $\tau$ is the lifetime constant, and $R=1 / \tau$ is the relaxation rate of fluorescent emission. In a simplified model of a fluorescence measurement, in which the noise due to the photodetector and ambient/stray light are neglected, the optical signal acquired by the photodetector $(S)$ is the sum of fluorescence $\left(s_{I}\right)$ and autofluorescence $\left(s_{2}\right)$

$$
S=s_{1}+s_{2}=s_{10} \exp \left(-R_{1} t\right)+s_{20} \exp \left(-R_{2} t\right)
$$

If the fluorescent contrast is defined as the fluorescence-to-autofluorescence ratio (FAR), one has

$$
F A R=\frac{s_{1}}{S_{2}}=\frac{s_{10}}{s_{20}} \exp \left[t\left(R_{2}-R_{1}\right)\right]
$$

For a given fluorophore and imaging condition, $s_{10}$ and $s_{20}$ are constant because autofluorescence is dictated by the excitation wavelength. If the fluorophore (e.g., QDs) has a longer lifetime constant than that of background tissue $\left(\tau_{1}>\tau_{2}\right)$, one has $R_{1}<R_{2}$. As a result, the FAR is exponentially amplified with increasing time-delay.

The excitation pulse impinges on the interface, undergoes scattering while traveling within the medium, and emerges as a broadened version of its original waveform. The characteristic of the broadening is described by the temporal point spread function. In the context of small animal imaging, the width of the broadened pulse is significantly shorter than the lifetime constants of QDs [18]. Therefore, if the time-delay of the optical acquisition is adjusted long enough, the contributions of the excitation light pulse and autofluorescence can be sufficiently suppressed to a point where the fluorescent signal can be acquired without a fluorescent filter. Because of the entire emission spectrum contributes to the fluorescent signal, the detection efficiency is significantly improved. In addition, due to the increased FAR, the fluorescent contrast can also be improved.

\subsection{Experimental setup}

The optical imaging platform consists of an ultrafast pulsed titanium:sapphire (Ti:sapphire) laser, an intensified chargecoupled devices (ICCD) camera, a galvanometer-based dual-axis optical scanner, and an animal rotation stage. The imaging platform was controlled using the LabVIEW (version 8.6, National Instruments, Austin, TX) program developed in our lab. A diagram of the imaging platform and its dataflow is shown in Fig. 1. The laser and the ICCD camera are positioned for transillumination measurement. The overall configuration is similar to the one described previously [19], except the extension of capability from the continuous-wave (CW) to the time-resolved measurements. Such extension was achieved by replacement of the CW diode laser with the Ti:sapphire ultrafast pulsed laser and addition of the image intensifier in front of the CCD camera. 


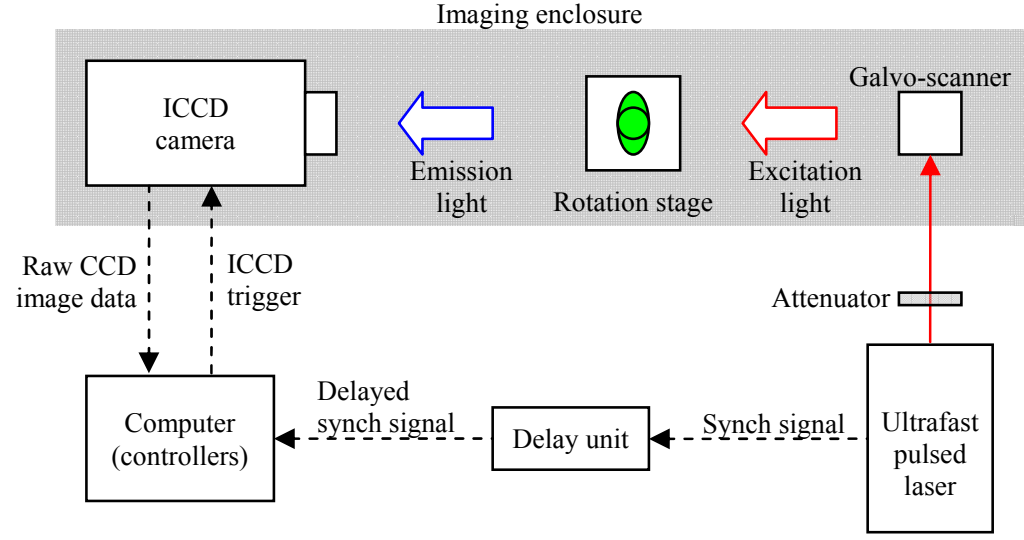

Fig. 1. Diagram of the time-resolved fluorescence tomography system. The light source (galvo-scanner) and the photodetector (ICCD camera) are arranged in a transillumination configuration with respect to the animal, which is vertically suspended on the rotation stage. The electrical synchronization signal from the pulsed laser is fed to the computer, where the ICCD controller is situated, via the time-delay unit. The components within the gray-colored area are enclosed by a light-tight imaging enclosure.

The Ti:sapphire laser (Chameleon Ultra, Coherent, Santa Clara, CA) produces a train of light pulses with a pulse width of $140 \mathrm{fs}$ and a repetition rate of $80 \mathrm{MHz}$, and has a spectral tuning range of 690 to $1040 \mathrm{~nm}$. In this study, we tuned the wavelength of the laser to $690 \mathrm{~nm}$. The average power of the laser beam was attenuated to $50 \mathrm{~mW}$ before it enters the imaging enclosure.

The control scheme of the imaging platform was also upgraded to accommodate time-resolved acquisition. A photodiode in the laser system samples its optical output and generates an electrical synchronization signal. This synchronization signal was used as the clock to control the timing between the laser and the ICCD camera.

Two imaging phantoms were used in this study $-\mathrm{a}$ thin rectangular container $(100 \times 100 \times 15 \mathrm{~mm})$ and a long cylindrical container $(22 \times 120 \mathrm{~mm})$, both filled with a liquid mixture of Intralipid (Fresenius Kabi, Bad Homburg, Germany) and India ink (off-the-shelf) in water. The optical properties of the liquid phantom at the excitation and the emission wavelengths are shown in Table 1. The fluorophore was polymer-coated QDs (Qtracker 800, Invitrogen, Carlsbad, CA) that have a fluorescent emission spectrum centered at $785 \mathrm{~nm}$. The concentration of the QDs was $50 \mathrm{nM}$ in de-ionized water. A QD inclusion was formed by injecting the QD solution in a small quartz sphere, which has an inner diameter of $3 \mathrm{~mm}$. This QD inclusion was suspended at the center inside the imaging phantoms using a thin and long acrylic rod.

Table 1. Optical properties of the phantom.

\begin{tabular}{|l|l|l|l|l|}
\hline $\boldsymbol{\lambda} \mathbf{( n m})$ & $\boldsymbol{\mu}_{a}\left(\mathbf{m m}^{-\mathbf{1}}\right)$ & $\boldsymbol{\mu}_{\boldsymbol{s}}^{\prime}\left(\mathbf{m m}^{\mathbf{- 1}}\right)$ & $\mathbf{g}$ & $\boldsymbol{n}$ \\
\hline 690 & 0.0079 & 1.62 & 0 & 1.37 \\
\hline 785 & 0.0104 & 1.13 & 0 & 1.37 \\
\hline
\end{tabular}

The study was divided into two experiments. In the first experiment, the rectangular phantom was used to characterize the temporal responses of the ICCD camera to the laser, the phantom, and the fluorescent inclusion. In addition, a euthanized nude mouse was used to demonstrate the similarity of the phantom and actual animal tissue. The temporal response of the ICCD camera was obtained by varying the time-delay between the optical acquisition and the reference synchronization signal. The width of the time-gates was 300 ps with a step size of 100 ps. In these time-resolved measurements, the laser firstly illuminated a glass diffuser (Thorlabs, Newton, NJ); secondly the imaging phantom without the QD inclusion; and finally the complete phantom. Acquisition of each of the time-series measurements was divided into several segments, each with appropriate intensifier gain to accommodate the limited dynamic range of the ICCD camera, 4095 due to a 12-bit analog/digital converter. Each segment had overlapping time points with its adjacent segments to allow equalization of gain after data acquisition.

To validate the proposed acquisition method, the impinging location of the laser on the phantom was about $10 \mathrm{~mm}$ away from the location of the QD inclusion, so that the patterns of excitation and emission would be sufficiently different. The same configuration was used for both of the time-gated and the fluorescent filter-based acquisition. In the time-gated acquisition, the gate width was $1 \mathrm{~ns}$ with a delay of $8 \mathrm{~ns}$. Note that a 750-nm long-pass filter was used to block the temporal residual light between laser pulses. In acquisition using a fluorescent filter (785-nm central wavelength with 
10-nm pass-band), the gate width was 1 ns without delay. Other parameters were identical for both acquisitions. The schematic of acquisition timing is illustrated in Fig. 2.

In the second experiment, the cylindrical phantom was used to demonstrate the proposed acquisition method under a relatively realistic condition for small animal fluorescence tomography. The QD inclusion was positioned at the center of the phantom. The phantom was mounted on the rotation stage, which changes the acquisition angle to allow full-angle sampling (in 20 angular steps). For each acquisition angle, 15 source positions were used, which were evenly distributed in a $3 \times 5$ grid with step sizes of $2.5 \mathrm{~mm}$ (horizontally) and $3.5 \mathrm{~mm}$ (vertically). The same parameters for the time-gate and the intensifier gain used in the previous experiment were applied during fluorescence measurements. The CCD integration time $(100 \mathrm{~ms})$ was the same in both the time-gated and the filter-based methods. The excitation data was measured without the fluorescent filter and using a gate width of $1 \mathrm{~ns}$ without delay.

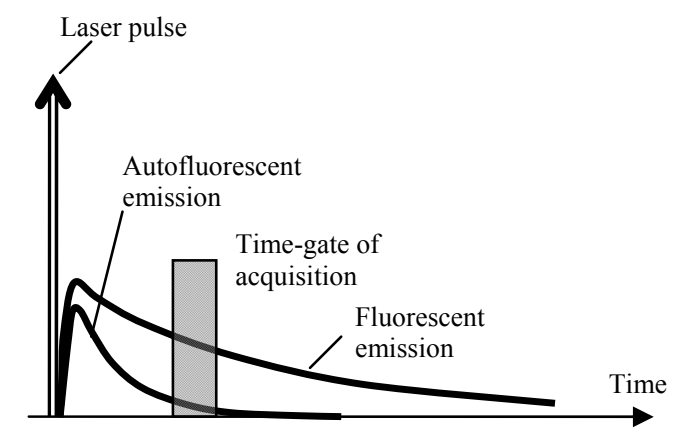

Fig. 2. Illustration of temporal responses of signals due to fluorescent and autofluorescent emissions in time-

\subsection{Image reconstruction}

The forward problem for image reconstruction was solved using our parallel Monte Carlo software. Because of the widely separated excitation and the emission wavelengths (690 and $785 \mathrm{~nm}$, respectively), the differences in optical properties for the excitation and the emission light have to be taken into account. The sensitivity matrix was obtained by explicitly computing the photon affluence rates for the sources (at the excitation wavelength) and the detectors (at the emission wavelength). For each simulation, 10 million simulated photons were used. The regularized inverse problem solver was the same as the one previously published, which used a conjugate gradient algorithm to minimize a three-term cost function that penalizes data fidelity, data size, and smoothness [20]. The same regularization parameters were used for both the time-gating and filter-based datasets. The voxel size was $(0.19 \mathrm{~mm})^{3}$ for the forward problem solver, and $(0.75 \mathrm{~mm})^{3}$ for the inverse problem solver. For each acquisition angle and each source position, 20 detector positions were used, which were evenly distributed on a $4 \times 5$ grid with step sizes of $3 \mathrm{~mm}$ horizontally and $4 \mathrm{~mm}$ vertically.

\section{RESULTS AND DISCUSSION}

The temporal responses of the ICCD camera to the ultrafast pulsed laser (glass diffuser), the nude mouse cadaver, the rectangular imaging phantom (without QD), and the QD inclusion (immersed in the phantom) were measured using a series of time-delays ranging from 0 to $12.5 \mathrm{~ns}$ (inverse of the laser repetition rate), Fig. 3. Note that these temporal responses were in fact spatial-temporal convolutions of the temporal response of the ICCD camera and the measured physical phenomena, as well as the spatial-temporal point spread function of the light waves within the medium. Because the goal was to qualitatively analyze these physical phenomena, and also because the gate-width was relatively narrow (300 ps) compared to the temporal scale that are interesting to this study (on the order of nanoseconds), the overall trends of the curves are treated the same as the actual temporal response in our analysis throughout this article unless otherwise specified.

Comparing the temporal responses of the QDs and the imaging phantom in Fig. 3, it is evident that the relative signal intensity of the QDs to that of the background (imaging phantom) increases as the time-delay increases. Note that the inter-pulse interval is $12.5 \mathrm{~ns}$ for the Ti:sapphire laser. It can be inferred from the curve of the phantom that the laser pulse arrives the photodetector at approximately $3000 \mathrm{ps}$, where the curve has an abrupt upward turn, which is consistent with the peak position of the laser (glass diffuser) response. The delay of approximately $1 \mathrm{~ns}$ between the laser and the phantom responses was due to the strong scattering within the phantom. In fact, it is well known that the amount of timedelay of a transmitted pulse is a good measure of average scattering of a bulk medium. 
By examining the temporal responses of the QDs and the imaging phantom, we chose a time-delay of $8 \mathrm{~ns}$ (from the arrival of the laser pulse) for the 2D time-gated acquisition. Fig. 4 is the comparison of $2 \mathrm{D}$ fluorescent measurements of the imaging phantom with QD inclusion using the time-gated and the filter-based acquisition methods. The bright-field image in Fig. 4(a) was obtained without filling the container with liquid phantom, which showed the exact position of the QD

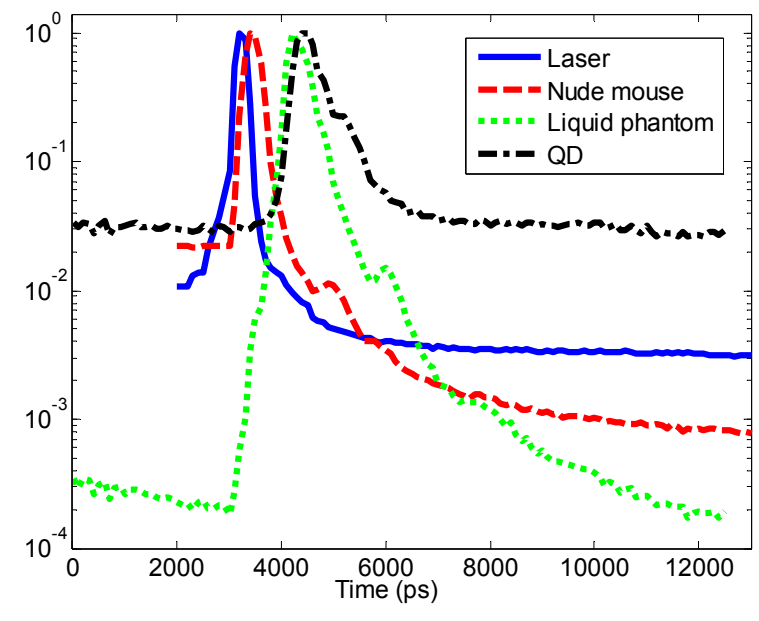

Fig. 3. Temporal responses of the ICCD camera (normalized to unity) to the ultrafast pulsed laser, a nude mouse, the liquid phantom (without the QD inclusion), and the QD inclusion immerged in the liquid phantom. The time-resolved acquisitions were performed using a gate width of $200 \mathrm{ps}$ and a step size of 100 ps. Note the dramatic increment of relative intensities of the QDs vs. that of the liquid phantom or the nude mouse as the time-delay increases. inclusions, as well as the impinging and exiting positions of the laser on the walls of the container. The image shown in Fig. 4(b) is the excitation light pattern, which was acquired without time-delay (but with a 750-nm long-pass filter in place to block residual light between laser pulses), which co-localized well with the impinging position of the laser. The fluorescent emission light patterns were acquired using the time-gated and filter-based methods with otherwise identical acquisition parameters, Figs. 4(c) and (d), respectively, which co-localizes with the position of the QD inclusion, i.e., the glass sphere in Fig. 4(a). The identical structure and position of the fluorescent patterns shown in Figs. 4(c) and (d) validated the effectiveness of the proposed acquisition method. Note that in the two fluorescent measurements, other acquisition parameters were identical: gate width of $1 \mathrm{~ns}, \mathrm{CCD}$ integration time of $100 \mathrm{~ms}$, and the intensifier voltage of $800 \mathrm{v}$. The signal intensity shown in the figures were in arbitrary unit.

In Fig. 4(e), line profiles of fluorescent images were plotted along a horizontal line passing the projection center of the QD inclusion, shown as white dashed broken lines in Figs. 4(c) and (d). Note that the fluorescent image acquired using the time-gated method produced significantly higher signal intensity (relative intensity of the two profiles measured 11.5 at the peaks). Because the signal intensity is linearly related to integration time of the ICCD camera, this increase in fluorescent signal intensity can be translated to a reduction of integration time by the same factor. It is well known that fluorescence tomography is limited by the amount of detectable fluorescent light. An increase of detection efficiency by a factor of $>10$ will dramatically improve image quality and enable more preclinical imaging applications.

Note that in a true continuous wave $(\mathrm{CW})$ measurement, the photodetector (such as a CCD camera) is on continuously, and acquires more photons than a time-gated detector. Therefore, the true performance of a filter-based method, which more likely uses CW measurement, is better than the one demonstrated in Fig. 4(d). In this study, the majority of the photons, calculated 56\% according to the QD curve in Fig. 3, are detected because the acquisition window was set at the peak of the fluorescent emission.

The performance of the proposed method in terms of autofluorescence suppression is shown in Fig. 4(f), where the line profiles of the excitation, and the two emission patters are plotted along the horizontal line passing the center of the excitation pattern, green dotted broken lines in Figs. 4(b-d). The line profiles of the two fluorescent patterns are identical on the right side of the curves. On the left side, however, the fluorescent pattern acquired using the filter-based method is consistently broader than using the time-gated method. Additional filter-based measurement using a band-pass filter with the same central wavelength but a broader pass-band and a significantly better stop-band rejection produced similar fluorescent line profiles (data not shown). This indicates that the broadening of the fluorescent line profile was due to autofluorescence rather than transmission of the excitation light (due to finite optical density of the fluorescent filter).

Tomographical reconstructions were performed on the fluorescent data acquired using the two methods. The two datasets produced almost identical results, both of which accurately reconstructed the position of the QD inclusion. The axial slices taken at the height of the QD inclusion are shown in Fig. 5 (results normalized to unity). The profiles along the horizontal line passing the center are also almost identical. The ability of the proposed method to produce the same artifact-free result as the standard method further validated the effectiveness of this proposed method. 

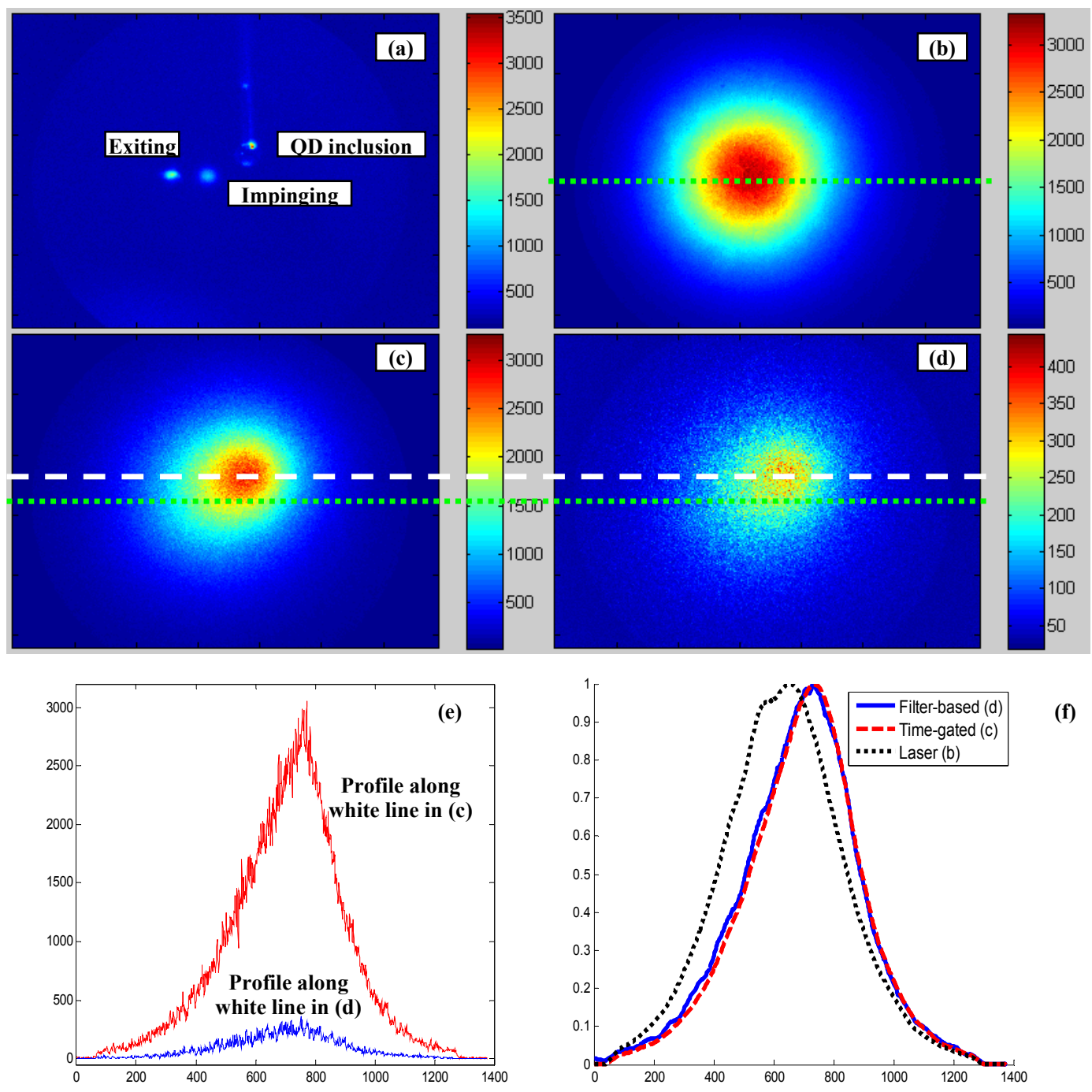

(f)

Fig. 4. Comparisons of 2D fluorescent measurements of QD inclusion in the rectangular phantom using the time-gated and ordinary BPF-based acquisition methods. (a) Bright-field image of the empty rectangular container shows the location of the QD inclusion and the impinging/exiting positions (bright spots to the left of the QD inclusion) of the laser on the rectangular phantom. (b) Excitation light pattern acquired without time-delay, which co-localized well with the impinging point of the laser on the phantom. (c) Fluorescent emission pattern acquired using the time-gated method with a time-delay of $8 \mathrm{~ns}$, which colocalized well with the location of the QD inclusion, i.e., the sphere shown in (a). (d) Fluorescent emission pattern acquired using a 785/10 nm BPF without time-delay. (e) Comparison of the fluorescent signal intensities along the dashed (white) lines in (c) and (d), red and blue, respectively, showed a ratio of 11.5 at the maxima of the curves. (f) Comparison of the signal intensities (smoothed and normalized) along the dotted (green) lines in (b-d), in which the filter-based signal appears broader than the time-gated on the left half of their profiles, indicating that the excitation light contributes less in the time-gated acquisition than that using a band-pass filter.

Admittedly, the absolute signal intensity decrease with increased time-delay, which can be largely compensated by a number of acquisition techniques (such as more intense excitation light, longer integration time, and higher gain of the image intensifier). Under most circumstances in fluorescence tomography, the benefits of higher contrast outweigh the lower signal intensity. 


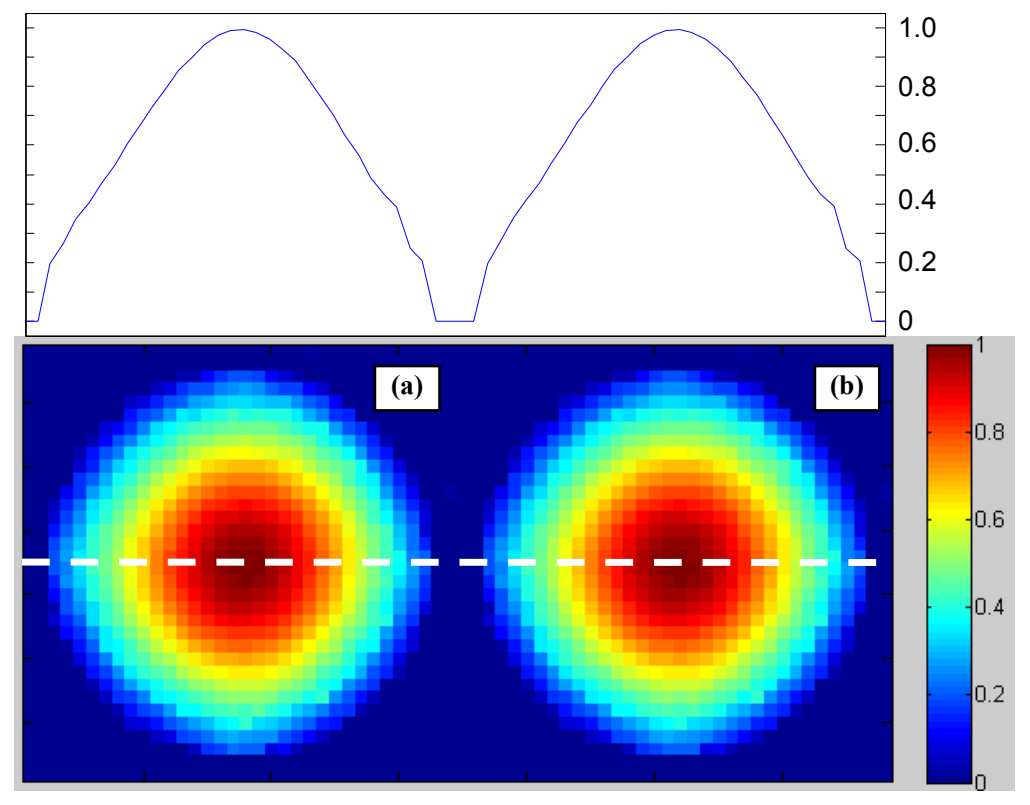

Fig. 5. Comparison of tomographic reconstructions of the QD inclusion in the cylindrical phantom using (a) the time-gated acquisition method (time-delay of $8 \mathrm{~ns}$ ), and (b) the fluorescent filter-based acquisition method (band-pass filter with 785$\mathrm{nm}$ central wavelength and $10-\mathrm{nm}$ pass-band).

Note that in both measurements, the gate width of the image intensifier was $1 \mathrm{~ns}$, the intensifier voltage was $750 \mathrm{v}$, and the CCD integration time was $320 \mathrm{~ms}$. The reconstruction results were normalized to unity for easier comparison. The line profiles of the reconstructions were drawn across the center of the cylinder, where the intensity was maximal.

Previous 2D imaging studies of QDs in liquid samples, cells, or subcutaneous tumors in animals have consistently shown that time-gating can effectively suppress autofluorescence [14-17]. In this study, liquid phantoms were used to demonstrate the proposed time-gated acquisition method for tomographic reconstruction. Because of the temporal responses of the phantom and nude mouse cadaver have similar characteristics, shown in Fig. 3, the proposed time-gated acquisition method has the potential to also produce favorable results in animal experiments in future in vivo studies.

\section{CONCLUSIONS}

We proposed a time-gated acquisition method for fluorescence tomography, which exploited the difference of temporal characteristics of the fluorophore (QDs in this study) and the background (tissue-simulating imaging phantom). Using this time-gated acquisition method, the need for band-pass fluorescent filter is eliminated. An immediate advantage of the proposed method was that the entire emission spectrum contributes to the fluorescent signal and thus significantly increased the efficiency of detection, a factor of 11.5 compared to using a typical $10-\mathrm{nm}$ pass-band fluorescent filter. In addition, this technique enables the use of nonstandard (e.g., lab-developed) or modified QDs that may not have matching filters available. Another advantage that makes the proposed technique attractive is its potential to suppress autofluorescence in animal experiments, which limits sensitivity in fluorescence tomography.

\section{ACKNOWLEDGEMENT}

This study was funded by NIH/NCRR P41 RR005959 and NIH/NCRR 1R21 RR025824.

\section{REFERENCES}

[1] Ntziachristos, V. Bremer, C. and Weissleder R., "Fluorescence imaging with near-infrared light: new technological advances that enable in vivo molecular imaging," Eur. Radiol. 13, 195-208 (2003).

[2] Massoud, T. F. and Gambhir S. S., "Molecular imaging in living subjects: seeing fundamental biological processes in a new light," Genes Dev., 17, 545-580 (2003).

[3] Ntziachristos, V., Ripoll, J., Wang, L. V. and Weissleder R., "Looking and listening to light: the evolution of wholebody photonic imaging," Nature Biotechnol., 23, 313-320 (2005). 
[4] Grimm, J., et. al., "Use of gene expression profiling to direct in vivo molecular imaging of lung cancer," Proc. Natl. Acad. Sci., 102, 14404-14409 (2005).

[5] Bloch, S., Lesage, F., McIntosh, L., Gandjbakhche, A., Liang, K. and Achilefu S., "Whole-body fluorescence lifetime imaging of a tumor-targeted near-infrared molecular probe in mice," J. Biomed. Opt., 10, 054003 (2005).

[6] Garofalakis, A., et. al., "Three-dimensional in vivo imaging of green fluorescent protein-expressing T cells in mice with noncontact fluorescence molecular tomography," Mol. Imaging, 6, 96-107 (2007).

[7] Michalet, X., et. al., "Quantum dots for live cells, in vivo imaging, and diagnostics," Science, 307, 538-544 (2005).

[8] Smith, A. M., Duan, H., Mohs, A. M. and Nie S., "Bioconjugated quantum dots for in vivo molecular and cellular imaging," Adv. Drug Deliv. Rev., 60, 1226-1240 (2008).

[9] Ballou, B., Lagerholm, B. C., Ernst, L. A., Bruchez, M. P. and Waggoner, A. S., "Noninvasive imaging of quantum dots in mice," Bioconjug. Chem., 15, 79-86 (2004).

[10] Morgan, N. Y., et. al., "Real time in vivo non-invasive optical imaging using near-infrared fluorescent quantum dots," Acad. Radiol., 12, 313-323 (2005).

[11] Texier, I. and Josser, V., "In vivo imaging of quantum dots," Methods Mol. Biol., 544, 393-406 (2009).

[12] Stylianos, P., Giannis, Z., Anikitos, G., Rosy, F. and Jorge, R., "Autofluorescence removal from fluorescence tomography data using multispectral imaging," Proc. SPIE, 6626, 66260I (2007).

[13] Soloviev, V. Y., et. al., "Fluorescence lifetime tomography of live cells expressing enhanced green fluorescent protein embedded in a scattering medium exhibiting background autofluorescence," Opt Lett., 32, 2034-2036 (2007).

[14] Dahan, M., et. al., "Time-gated biological imaging by use of colloidal quantum dots," Opt Lett., 26, 825-827 (2001).

[15] Mitchell, A. C., Dad, S. and Morgan, C. G., "Selective detection of luminescence from semiconductor quantum dots by nanosecond time-gated imaging with a colour-masked CCD detector," J. Microscopy, 230, 172-176 (2008).

[16] Giraud, G., et. al., "Fluorescence lifetime imaging of quantum dot labeled DNA microarrays," Intl. J. Mol. Sci., 10, 1930-1941 (2009).

[17] May, A., Bhaumik, S., Gambhir, S. S., Zhan, C. and Yazdanfar, S., "Whole-body, real-time preclinical imaging of quantum dot fluorescence with time-gated detection," J. Biomed. Opt., 14, 060504 (2009).

[18] Schlegel, G., Bohnenberger, J., Potapova, I. and Mews, A., "Fluorescence decay time of single semiconductor nanocrystals," Phys. Rev. Lett., 88, 137401 (2002).

[19] Zhang, X., Badea, C. T. and Johnson, G. A., "Three-dimensional reconstruction in free-space whole-body fluorescence tomography of mice using optically reconstructed surface and atlas anatomy," J. Biomed. Opt., 14, 064010 (2009).

[20] Zhang, X. and Badea, C. T., "Effects of sampling strategy on image quality in noncontact panoramic fluorescence diffuse optical tomography for small animal imaging," Opt. Express, 17, 5125-5138 (2009). 